\title{
Psychological factors influence the gastroesophageal reflux disease (GERD) and their effect on quality of life among firefighters in South Korea
}

\section{Seung-Ho Jang ${ }^{1}$, Han-Seung Ryu ${ }^{2}$, Suck-Chei Choi ${ }^{2}$, Sang-Yeol Lee ${ }^{1}$}

\author{
${ }^{1}$ Department of Psychiatry, School of Medicine, Wonkwang University, Iksan, Republic of Korea, ${ }^{2}$ Division of \\ Gastroenterology, Department of Internal Medicine, School of Medicine, Wonkwang University, Iksan, Republic \\ of Korea
}

Objectives: The purpose of this study was to examine psychosocial factors related to gastroesophageal reflux disease (GERD) and their effects on quality of life (QOL) in firefighters.

Methods: Data were collected from 1217 firefighters in a Korean province. We measured psychological symptoms using the scale. In order to observe the influence of the high-risk group on occupational stress, we conduct logistic multiple linear regression. The correlation between psychological factors and QOL was also analyzed and performed a hierarchical regression analysis.

Results: GERD was observed in $32.2 \%$ of subjects. Subjects with GERD showed higher depressive symptom, anxiety and occupational stress scores, and lower self-esteem and QOL scores relative to those observed in GERD - negative subject. GERD risk was higher for the following occupational stress subcategories: job demand, lack of reward, interpersonal conflict, and occupational climate. The stepwise regression analysis showed that depressive symptoms, occupational stress, self-esteem, and anxiety were the best predictors of QOL.

Conclusions: The results suggest that psychological and medical approaches should be combined in GERD assessment.

Keywords: Gastoesophageal reflux disease, Firefighters, Depressive symptoms, Anxiety, Occupational stress, Quality of life, Korea

\section{Introduction}

In South Korea, firefighters are civil servants whose job duties involve emergency rescue, fire prevention, fire vigilance, and fire extinguishment. Considering the systematic functional aspects of their duties, they can be classified into safety and volunteer functions. Moreover, firefighting duties have special characteristics in terms of risk, 24-h shifts, and working environments, relative to the duties of other civil servants. ${ }^{1}$ In addition, as firefighters face health-related risks and emergencies and work in uncommon environments, they are required to maintain physical fitness. Although firefighting duties differ according to type and characteristics, fire extinguishment activities involve life-threatening risks, such as those involving exposure to toxic gases including carbon monoxide and phosgene, death or injury, and accidents caused by unpredicted explosions. Moreover, firefighters are required to act promptly in both extinguishment and rescue and first aid activities to avoid risk to survivors. Therefore, firefighters are exposed to various serious risks; in addition,

Correspondence to: Sang-Yeol Lee, Department of Psychiatry, Schoo of Medicine, Wonkwang University, 895 Muwangro, Iksan 570-711, Jeollabukdo, Republic of Korea. Email: psysangyeol@hanmail.net the risk of the development of emotional stress and diseases resulting from their duties is high. ${ }^{2}$

Nevertheless, studies examining exposure risk in working environments and the biological monitoring of firefighters are rare, and the topics of studies examining the effects of firefighters' duties on their health are limited to occupational stress; post-traumatic stress disorder; and physical diseases including pulmonary dysfunction, respiratory symptoms, noise-induced deafness, musculoskeletal diseases, and cancer. ${ }^{3-5}$ One recent study reported that the prevalence of functional gastrointestinal disorders was very high in firefighters. ${ }^{6}$ In particular, gastroesophageal reflux disease (GERD) caused by the irregular working hours and a work structure involving high stress imposes a serious burden on firefighters' health.

GERD patients have reported the following symptoms: GERD induced physical complications (reflux esophagitis and its complications and reflux-related respiratory and laryngopharyngeal complications), or in the absence of these complications, disorders that influence health and health-related quality of life (QOL) in a clinically significant way. ${ }^{7}$ Reflux-related symptoms include typical (heartburn and reflux) and atypical 
(chest pain, dysphagia, dyspepsia, asthma, bronchitis, and pneumonia) reflux symptoms. ${ }^{8}$ Many studies have defined GERD according to symptom frequency; the Rome III criteria recently reported that the most frequently used criterion for GERD diagnosis is the occurrence of GERD symptoms more than once per week. The reported prevalence of GERD has increased recently in Asia., ${ }^{9,10}$ The prevalence of GERD in South Korea exceeds $5 \% .^{11}$

Various psychosocial factors, including chronic stress, emotional instability, abnormal acid reflux, and obesity, are associated with GERD manifestation and symptoms. ${ }^{12,13}$ In particular, emotional instability, including depression and anxiety, is associated with increased risk of GERD. ${ }^{14}$ The effects of functional gastrointestinal disorders, including irritable bowel syndrome, on self-esteem have been reported in previous studies. ${ }^{15}$ However, the effects of GERD have not been examined. QOL in GERD patients is similar to that observed in patients with diabetes mellitus, cancer, and ischemic heart disease and lower relative to that of patients with duodenal ulcer, hypertension, heart failure, and menopause. ${ }^{16,17}$

Although the prevalence of GERD is high in firefighters and psychological factors are associated with GERD manifestation and exacerbation, studies examining these relationships are rare. This study explored psychological factors related to GERD and their effects on QOL in firefighters working in South Korea.

\section{Methods}

\section{Subjects}

Data were collected between September and October 2014. In total 1355 firefighters participated in the study. The definition and categorization of GERD was based on the Montreal criteria. ${ }^{7}$ The exclusion criteria included (a) esophageal or gastric malignancy; (b) previous gastric surgery; (c) peptic ulcer disease; (d) use of chronic antiacid medication, such as proton pump inhibitors or $\mathrm{H}_{2}$-receptor antagonists for more than two months prior to the survey and (e) pregnancy. Data for 1,217 subjects were included in the analysis (eligibility rate $=89.81 \%$ ). All study subjects provided written informed consent. This study was approved by the Wonkwang University Hospital Institutional Review Board (IRB \#. 1564).

\section{Measures}

\section{Patient health questionnaire-9}

The Patient Health Questionnaire-9 (PHQ-9) consists of nine items used to measure depression. ${ }^{18}$ Subjects provide responses regarding the severity of depression experienced in the preceding two weeks, with each item awarded 0-3 points. Overall scores of 5-9, 10-14, and $\geq 15$ indicate mild, moderate, and severe depression, respectively. The validity and reliability of the scale have been demonstrated in a Korean sample (Cronbach's $\alpha=0.88){ }^{19}$

\section{Generalized anxiety disorder questionnaire-7}

TheGeneralizedAnxiety DisorderQuestionnaire-7(GAD-7) consists of seven items, used to identify anxiety. Each item is awarded $0-3$ points, and the maximum score is $21 .{ }^{20}$ The sensitivity and specificity of the scale are highest with a cutoff score of 8 , and a cutoff score of 10 is optimal for detecting anxiety. ${ }^{21}$ Cronbach's $\alpha$ for the scale was high (0.98) in the current study.

\section{Korean occupational stress scale}

The Korean Occupational Stress Scale (KOSS) was developed to assess occupational stress and consists of eight domains: physical environment, job demand, insufficient job control, interpersonal conflict, job insecurity, organizational system, lack of reward, and occupational climate. ${ }^{22}$ We used the KOSS and the Korea Occupational Safety \& Health Agency Guide H-67-2012, to obtain occupational stress scores. We multiplied the scores for each subcategory by 100 . The high-scoring group (above the median score for Korean workers) was defined as the high-risk stress group, and the low-scoring group (below the median score for Korean workers) was defined as the low-risk stress group. ${ }^{23}$

\section{Rosenberg's self-esteem scale}

Rosenberg's Self-Esteem Scale (RSES) consists of ten items, five worded positively and five worded negatively. Responses are provided using a four-point scale ranging from 1 = ("strongly disagree") to 4 = ("strongly agree"), with higher scores indicating higher self-esteem. Total possible RSES scores range from 10 to $40 .^{24}$ The validity and reliability of the scale have been demonstrated in a Korean sample. ${ }^{25}$ Cronbach's $\alpha$ was high $(0.87)$ in the current study.

\section{World health organization QOL-BREF}

The World Health Organization QOL-BREF (WHOQOL-BREF) is a QOL scale and consists of five domains (overall QOL and general health, physical health, psychological health, social relationship, and environment). Each item is awarded a score of 0-5, and higher scores indicate higher QOL. ${ }^{26}$ The validity and reliability of the scale have been demonstrated in a Korean sample (Cronbach's $\alpha=0.92) .{ }^{27}$

\section{Statistical Analysis}

We calculated means and standard deviations for continuous variables and frequencies and percentages for categorical variables. We performed $\chi^{2}$ tests to analyze differences in demographic characteristics between subjects with and without GERD (GERD-positive and-negative groups, respectively). Independent $t$-tests were performed to analyze total PHQ-9, GAD-7, KOSS, RSES, and WHOQOL-BREF scores as continuous variables. We assessed GERD risk for each of the KOSS sub-categories via logistic regression analysis, after controlling for sex, 
Table 1 Demographic characteristics of subjects

\begin{tabular}{|c|c|c|c|c|c|}
\hline Variables & & GERD-positive $N=392 ; N(\%)$ & GERD-negative $N=825 ; N(\%)$ & $\chi^{2}$ & $P$ \\
\hline \multirow[t]{2}{*}{ Sex } & $\mathrm{M}$ & $365(93.1)$ & 775 (93.9) & 0.307 & 0.580 \\
\hline & $\mathrm{F}$ & $27(6.9)$ & $50(6.1)$ & & \\
\hline \multirow[t]{4}{*}{ Age(years) } & $<30$ & $15(3.8)$ & $48(5.8)$ & 11.748 & 0.008 \\
\hline & 30-39 & $114(29.1)$ & $301(36.4)$ & & \\
\hline & $40-49$ & $184(46.9)$ & $353(42.7)$ & & \\
\hline & $\geq 50$ & $79(20.2)$ & $123(15.1)$ & & \\
\hline \multirow[t]{3}{*}{ Education (years) } & Low $(<10)$ & $6(1.5)$ & $13(1.5)$ & 0.308 & 0.959 \\
\hline & Middle (10-13) & $101(25.7)$ & $219(26.5)$ & & \\
\hline & High (>13) & $285(72.8)$ & $593(72.0)$ & & \\
\hline \multirow[t]{4}{*}{ Marital status } & With spouse & $329(83.9)$ & $674(81.6)$ & 4.485 & 0.214 \\
\hline & Single & $57(14.5)$ & $142(17.2)$ & & \\
\hline & Divorced & $6(1.6)$ & $6(0.8)$ & & \\
\hline & Others & $0(0.0)$ & $3(0.4)$ & & \\
\hline \multirow[t]{4}{*}{ Working period (years) } & $<3$ & $53(13.5)$ & $151(18.3)$ & 10.647 & 0.014 \\
\hline & $4-7$ & $32(8.1)$ & 101 (12.2) & & \\
\hline & $8-11$ & 76 (19.3) & $147(17.8)$ & & \\
\hline & $\geq 12$ & $231(59.1)$ & $426(51.7)$ & & \\
\hline \multirow[t]{4}{*}{ Task } & EMS & $97(24.7)^{\prime}$ & $200(24.2)$ & 0.328 & 0.955 \\
\hline & Rescue & $40(10.2)$ & $80(9.7)$ & & \\
\hline & Firefighting & $216(55.1)$ & $455(55.2)$ & & \\
\hline & Administration & $39(10.0)$ & $90(10.9)$ & & \\
\hline \multirow[t]{2}{*}{ Working pattern } & Shift work & $342(87.2)$ & $697(84.5)$ & 1.621 & 0.203 \\
\hline & Daytime work & $50(12.8)$ & $128(15.5)$ & & \\
\hline
\end{tabular}

Note: M: male, F: female, N: number, GERD: gastroesophageal reflux disease, EMS: emergency medical service.

age, and education. Predictive variables for QOL were explored using stepwise multiple regression analysis. Statistical analyses were performed using SPSS software (SPSS, Version 21; Chicago, IL, United States).

\section{Results \\ Difference between the GERD-positive and- negative groups}

GERD was observed in $32.2 \%$ of the firefighters. The results of the $\chi^{2}$ tests, conducted to examine demographic characteristics showed that age $\left(\chi^{2}=11.748\right.$, $\mathrm{d} f=3, \mathrm{P}=0.008)$ and working period $\left(\chi^{2}=10.647, \mathrm{~d} f=3\right.$, $\mathrm{P}=0.014$ ) differed significantly between the firefighters with and without GERD. However, there were no significant between-group differences in sex, education, marital status, task, or working pattern (Table 1). The results of the independent $t$-tests, conducted to identify between-group differences in psychological variables, showed that the presence of GERD differed significantly between groups. PHQ-9 $(t=-15.468, \mathrm{P}<0.001)$, GAD-7 $(t=-11.808$, $\mathrm{P}<0.001), \operatorname{KOSS}(t=-8.174, \mathrm{P}<0.001), \operatorname{RSES}(t=4.557$, $\mathrm{P}<0.001)$, and WHOQOL-BREF $(t=11.011, \mathrm{P}<0.001)$ scores all differed significantly between the two groups (Table 2).

\section{Age adjusted odd ratios for GERD according to psychological variables}

We performed logistic multiple linear regression analysis, age adjusted to minimize misinterpretation of the results. In the high-risk group, GERD risk for the following KOSS subcategories was significantly higher relative to that observed for other subcategories: job demand (OR: 1.83, 95\% CI: 1.34-2.51), interpersonal conflict (OR: $2.07,95 \%$ CI: 1.06-3.51), lack of reward (OR: 2.17, 95\% CI: 1.21-3.88), and occupational climate (OR: 1.49, 95\% CI: 1.09-2.02). No significant associations were observed between other subcategories (Table 3).

\section{Bivariate associations between predictor variables and QOL in the GERD-positive group}

In the GERD-positive group, QOL was positively correlated with RSES scores and negatively correlated with PHQ-9, GAD-7, and KOSS scores. Age showed a tendency toward negative correlation, while education showed a tendency toward positive correlation (age, $r=-0.129, \mathrm{P}<0.05$; education, $r=0.019, \mathrm{P}<0.05$; Table 4).

\section{Factors influencing QOL in the GERD-positive group}

Hierarchical multiple regression analysis was conducted to identify models that could predict QOL in GERD patients. We included task, which was a demographic variable expected to exert a significant influence on QOL, and variables that were significantly correlated with QOL in the previously conducted bivariate analysis (age; education; and PHQ-9, GAD-7, KOSS, and RSES scores), in the multiple regression analysis. In the final regression model, depression $(\beta=-0.345, \mathrm{P}<0.001)$, occupational stress $(\beta=-0.296, \mathrm{P}<0.001)$, self-esteem $(\beta=0.192, \mathrm{P}<0.001)$, and anxiety $(\beta=-0.099, \mathrm{P}=0.043)$ were included and explained $45.7 \%$ of the variance in overall QOL (Table 5).

\section{Discussion}

As a result of various psychosocial factors, firefighters are at high risk of experiencing psychological and physical diseases. Moreover, psychosocial factors are very closely associated with functional gastrointestinal disorders, including GERD. ${ }^{28}$ 
Table 2 Comparison of psychological variables between GERD-positive and GERD-negative group

\begin{tabular}{lccrc}
\hline & GERD-positive $(\boldsymbol{N}=\mathbf{3 9 2})$ & GERD-negative $(\boldsymbol{N}=\mathbf{8 2 5})$ & & \multicolumn{1}{c}{$\boldsymbol{t}$} \\
\cline { 2 - 3 } & $\mathrm{M} \pm \mathrm{SD}$ & $\mathrm{M} \pm \mathrm{SD}$ & -15.468 & $<0.001$ \\
\hline PHQ-9 & $8.17 \pm 5.72$ & $3.53 \pm 4.44$ & -11.808 & $<0.001$ \\
GAD-7 & $5.36 \pm 4.51$ & $2.43 \pm 3.77$ & -8.174 & $<0.001$ \\
KOSS & $65.47 \pm 8.02$ & $61.08 \pm 9.09$ & 4.557 & $<0.001$ \\
RSES & $75.49 \pm 18.87$ & $81.14 \pm 20.72$ & 11.011 & $<0.001$ \\
WHOQOL-BREF & $69.82 \pm 11.83$ & $78.61 \pm 13.54$ & \\
\hline
\end{tabular}

Note: M: mean, SD: standard deviation, N: number, GERD: gastroesophageal reflux disease, PHQ-9: Patient health questionnaire-9, GAD7: Generalized anxiety disorder questionnaire-7, KOSS: Korean occupational stress scale, RSES: Rosenberg's self-esteem scale, WHOQOL-BREF: World health organization quality of life-bref.

Table 3 Adjusted odds ratio of GERD according to occupational stress

\begin{tabular}{|c|c|c|c|c|c|}
\hline & & $\beta$ & OR & 95\% C.I. & $\mathbf{P}$ \\
\hline \multirow[t]{2}{*}{ Physical environment } & Low risk & & 1.0 & & \\
\hline & High risk & 0.141 & 1.15 & $0.83-1.59$ & 0.398 \\
\hline \multirow[t]{2}{*}{ Job demand } & Low risk & & 1.0 & & \\
\hline & High risk & 0.606 & 1.83 & $1.34-2.51$ & $<0.001$ \\
\hline \multirow{2}{*}{ Insufficient job control } & Low risk & & 1.0 & & \\
\hline & High risk & 0.033 & 1.03 & $0.65-1.66$ & 0.891 \\
\hline \multirow{2}{*}{ Interpersonal conflict } & Low risk & & 1.0 & & \\
\hline & High risk & 0.770 & 2.07 & $1.06-3.51$ & 0.010 \\
\hline \multirow[t]{2}{*}{ Job insecurity } & Low risk & & 1.0 & & \\
\hline & High risk & 0.386 & 1.47 & $0.68-3.17$ & 0.326 \\
\hline \multirow[t]{2}{*}{ Organizational system } & Low risk & & 1.0 & & \\
\hline & High risk & -0.200 & 0.82 & $0.51-1.31$ & 0.400 \\
\hline \multirow[t]{2}{*}{ Lack of reward } & Low risk & & 1.0 & & \\
\hline & High risk & 0.775 & 2.17 & $1.21-3.88$ & 0.009 \\
\hline \multirow[t]{2}{*}{ Occupational climate } & Low risk & & 1.0 & & \\
\hline & High risk & 0.396 & 1.49 & $1.09-2.02$ & 0.011 \\
\hline
\end{tabular}

Note: GERD: gastroesophageal reflux disease.

Table 4 Bivariate associations between predictor variables and quality of life in GERD group ( $N=392)$

\begin{tabular}{|c|c|c|c|c|c|c|c|c|c|}
\hline & 1 & 2 & 3 & 4 & 5 & 6 & 7 & 8 & 9 \\
\hline Quality of life & 1 & & & & & & & & \\
\hline Age & -0.129 & 1 & & & & & & & \\
\hline Education & $0.109^{*}$ & $-0.301^{* *}$ & 1 & & & & & & \\
\hline Marital status & -0.020 & $-0.415^{* *}$ & 0.024 & 1 & & & & & \\
\hline Working period & -0.080 & $0.765^{* *}$ & $-0.255^{*}$ & $-0.551^{* *}$ & 1 & & & & \\
\hline PHQ-9 & $-0.569^{* *}$ & 0.055 & -0.092 & 0.074 & 0.012 & 1 & & & \\
\hline GAD-7 & -0.436 & $0.119^{*}$ & $-0.101^{*}$ & 0.039 & 0.056 & $0.639^{* *}$ & 1 & & \\
\hline KOSS & $-0.471^{* *}$ & 0.053 & 0.037 & -0.019 & 0.028 & $0.457^{* *}$ & $0.385^{* *}$ & 1 & \\
\hline RSES & $0.450^{* *}$ & $-0.212^{* *}$ & 0.179 & 0.084 & $-0.190^{* *}$ & -0.248 & $-0.144^{* *}$ & $-0.281^{1 *}$ & 1 \\
\hline
\end{tabular}

Note: GERD: gastroesophageal reflux disease, 1: Quality of life, 2: Age, 3: Education, 4: Marital status, 5: Working period, 6: PHQ-9, 7: GAD-7, 8: KOSS, 9: RSES.'P $<0.05 .{ }^{*} P<0.01$.

Table 5 Factors influencing quality of life in GERD group ( $N=392)$

\begin{tabular}{lcccccc}
\hline Factors & Standardized $\beta$ & $\boldsymbol{t}$ & $\mathbf{P}$ & Adjusted $\boldsymbol{R}^{\mathbf{2}}$ & $\boldsymbol{F}$ & $\mathbf{P}$ \\
\hline PHQ-9 & -.345 & -6.726 & $<0.001$ & 0.457 & 83.308 & $<0.001$ \\
KOSS & -.296 & -7.533 & $<0.001$ & & & \\
RSES & 0.192 & 4.440 & $<0.001$ & & & \\
GAD-7 & -0.099 & -2.028 & 0.043 & & & \\
\hline
\end{tabular}

Note: GERD: gastroesophageal reflux disease, PHQ-9: Patient health questionnaire-9, GAD-7: Generalized anxiety disorder questionnaire-7, KOSS: Korean occupational stress scale, RSES: Rosenberg's self-esteem scale.

GERD was observed in 392 (32.2\%) firefighters. This prevalence is much higher relative to those reported in previous studies involving healthy populations..$^{29-31}$ Moreover, the prevalence of GERD differed according to age and duration of work. Previous studies showed that excessive work and irregular working hours affected firefighters' psychological and physical diseases. ${ }^{32-34}$ Despite the high observed prevalence of GERD, GERD manifestation did not differ by demographic data, with the exception of age and working period, suggesting that psychosocial factors exerted a strong influence on GERD; in addition, a previous study reported that GERD prevalence increased with age. ${ }^{35}$

In comparison, significant differences in psychosocial factors, including depression, anxiety, occupational stress, 
self-esteem, and QOL, were observed between the GERDpositive and GERD-negative groups. In a previous study conducted by Jansson ${ }^{36}$ people with repetitive GERD were more likely to experience depression and anxiety relative to other people. In a community-based study, Hartono reported that depression, anxiety, and chronic stress led to GERD.$^{37}$ In addition, Martin-Merino reported that the odds ratio (OR) for GERD increased by $72 \%$ in people with depression, indicating a close relationship between GERD and psychosocial factors. ${ }^{38}$ No studies have been conducted to examine the effects of self-esteem on GERD. However, Lee et al. reported that self-esteem could relieve psychological symptoms such as depression or anxiety, resulting from occupational stress. Similarly, self-esteem could be expected to relieve psychological symptoms as a mediator in GERD, showing secondary effects. ${ }^{39}$ Two main theories have been used to explain the relationships between psychosocial factors and GERD. First, GERD could cause secondary depression and anxiety, increasing sensitivity to GERD. Second, genetic sensitivity to GERD is much higher in people who are susceptible to psychosocial factors. Therefore, various factors are involved in the relationships between psychosocial factors and GERD, and it is difficult to define them via a single model. ${ }^{40} \mathrm{In}$ terms of context, the findings of this study are consistent with those of previous studies; moreover, by examining differences in occupational stress, self-esteem, and stress coping style, which had not been explored previously, the present study broadened the scope of understanding regarding the role of psychosocial factors in GERD.

The high-risk group was more likely to experience GERD associated with job demand, interpersonal conflict, lack of reward, and occupational climate. In addition, previous studies have reported that GERD was closely associated with stress. Rubin reported that emotional stress was strongly correlated with reductions in esophageal motility. ${ }^{41}$ Moreover, Baker reported that normal gastric acid secretion can be mistaken for GERD in people exposed to high levels of long-term stress. ${ }^{42}$ With regard to the stress subdomains, Wallander reported that high GERD risk was observed for occupational stressors including job demand, low job control, and job strain..$^{43}$ Moreover, self-imposed and time-related pressure were significantly correlated with GERD. In addition to firefighters' occupational characteristics, stress resulting from interpersonal conflict and occupational climate exerted a substantial influence on GERD, and could have been affected by hierarchical organization and a public service system focused on command and discipline, which form Korean occupational environments that differ markedly from those in Western countries.

Depression, occupational stress, self-esteem, and anxiety explained $45.7 \%$ of the variance in overall QOL and influenced QOL in the GERD-positive group. In addition to reflux-related symptoms, depression and anxiety influence QOL in GERD patients. Masoumi evaluated health-related QOL using the SF-36 questionnaire and found that GERD patients showed greater reductions in QOL in all areas, relative to those observed for the control group. ${ }^{44}$ Moreover, in Kovács's study, continuous everyday pressure was suggested to reduce QOL in GERD patients with depression and anxiety. ${ }^{45}$ Although QOL differed according to the presence of GERD in previous studies, they did not identify important psychosocial factors in this regard; therefore, the findings of the present study are significant.

The study had limitations. First, it was a cross-sectional study; therefore, we could not assess the effects of time. Second, we used self-report scales to evaluate psychological variables increasing the possibility of subjective bias, and the severity of GERD symptoms was not examined. Third, as the study was population based, it did not include endoscopic data; therefore, we could not determine the involvement of erosive esophagitis, non-erosive reflux disease, or functional heartburn. Fourth, because the study included only firefighters, who constitute a specific occupational group, generalization of the results to all GERD patients is limited. Fifth, as the proportion of male subjects in the study was large (94\%), we could not stratify by sex.

Despite these limitations, the study included a large number of GERD-positive subjects and examined the effects of occupational stress, stress coping, and self-esteem, which had not been previously examined. In addition, the study was significant, as it suggested a new approach to improving firefighters' physical and psychological health, which has gained attention as a social issue. In the future, the effects of various psychosocial factors on GERD should be examined and medical and psychiatric approaches should be used simultaneously for treatment.

\section{Disclosure statement}

No potential conflict of interest was reported by the authors.

\section{Funding}

This article was supported by Wonkwang University in 2016.

\section{References}

1 Ha JH, Kim DI, Seo BS, Kim WS, Yoo SH, Kim SK. Job stress and psychosocial stress among firefighters. Korean J Occup Environ Med. 2008;20:104-11.

$2 \mathrm{Kim}$ KS. Health hazards in firefighters. Hanyang Med Rev. 2010;30:296-304.

$3 \mathrm{Kim}$ GH, Kim JW, Kim SH. Influences of job stressors on psychosocial well-being, fatigue and sleep sufficiency among firefighters. Korean J Occup Environ Med. 2006;18:232-45.

4 Kim DS, Moon MK, Kim KS. A survey musculoskeletal symptoms and risk factors for the 119 emergency medical service (EMS) activities. J Ergon Soc Korea. 2010;29:211-6.

5 Lovejoy S, Gillespie GL, Christianson J. Exploring physical health in a sample of firefighters. Workplace Health Saf. 2015;63:253-8.

6 Roh SG, Kim JH. Work related diseases in Korean fire fighters. J Dis Pol Manage. 2013;11(10):571-6. 
7 Vakil N, van Zanten SV, Kahrilas P, Dent J, Jones R. The montreal definition and classification of gastroesophageal reflux disease: a global evidence-based consensus. Am J Gastroenterol. 2006;101:1900-20.

8 Dent J. Definition of reflux disease and its separation from dyspepsia. Gut. 2002;50:17-20.

9 Goh KL, Wong HT, Lim CH, Rosaida MS. Time trends in peptic ulcer, erosive reflux esophagitis, gastric and esophageal cancers in a multiracial Asian population. Aliment Pharmacol Ther. 2009;29:774-80.

10 Lien HC, Chang CS, Yeh HZ. Increasing prevalence of erosive esophagitis among Taiwanese aged 40 years and above: a comparison between two time periods. J Clin Gastroenterol. 2009;43:926-32.

$11 \mathrm{Kim}$ JI, Kim SG, Kim N. Changing prevalence of upper gastrointestinal disease in 28893 Koreans from 1995 to 2005. Eur J Gastroenterol Hepatol. 2009;21:787-93.

12 Jansson C, Nordenstedt H, Wallander MA, Johansson S, Johnsen R, Hveem $\mathrm{K}$, et al. Severe gastroesophageal reflux symptoms in relation to anxiety, depression and coping in a population-based study. Aliment Pharmacol Ther. 2007;26:683-91.

13 Johnston BT, Gunning J, Lewis SA. Health care seeking by heartburn sufferers is associated with psychosocial factors. Am J Gastroenterol. 1996;91:2500-4.

14 Hampel H, Abraham NS, El-Serag HB. Meta-analysis: obesity and the risk for gastroesophageal reflux disease and its complications. Ann Intern Med. 2005;143:199-211.

15 Tominaga K, Higuchi K, Iketani T, Ochi M, Kadouchi K, Tanigawa $\mathrm{T}$, et al. Comparison of gastrointestinal symptoms and psychological factors of functional dyspepsia to peptic ulcer or panic disorder patients. Inflammopharmacology. 2007;15(2):84-9.

16 Hongo M, Kinoshita Y, Shimozuma K, Kumagai Y, Sawada M, Nii M. Psychometric validation of the Japanese translation of the quality of life in reflux and dyspepsia questionnaire in patients with heartburn. J Gastroenterol. 2007;42:807-15.

17 Sjoland H, Wiklund I, Caidahl K, Haglid M, Westberg S, Herlitz J. Improvement in quality of life and exercise capacity after coronary bypass surgery. Arch Intern Med. 1996;156:265-71.

18 Kroenke K, Spitzer RL, Williams JB. The PHQ-9: validity of a brief depression severity measure. J Gen Intern Med. 2001;16:606-13.

19 Han C, Jo SA, Kwak JH, Pae CU, Steffens D, Jo I. Validation of the patient health questionnaire-9 Korean version in the elderly population: the Ansan Geriatric study. Compr Psychiatry. 2008;49:218-23.

20 Spitzer RL, Kroenke K, Williams JBW, Löwe B. A brief measure for assessing generalized anxiety disorder: the GAD-7. Arch Intern Med. 2006;166:1092-7.

21 Kroenke K, Spitzer RL, Williams JBW, Monahan PO, Löwe B. Anxiety disorders in primary care: prevalence, impairment, comorbidity, and detection. Ann Intern Med. 2007;146:317-25.

22 Chang SJ, Koh SB, Kang D, Kim SA, Kang MG, Lee CG. Developing an occupational stress scale for Korean employees. Korean J Occup Environ Med. 2005;17:297-317.

23 KOSHA. KOSHA GUIDE H-67-1012. Seoul: Korea Occupational Safety \& Health Agency; 2012.

24 Rosenberg HS. Society and adolescent self-image. Princeton (NJ): Princeton University Press; 1965.

25 Lee JY, Nam SK, Lee MK, Lee JH, Lee SM. Rosenberg`self-esteem scale: analysis of item-level validity. Korean J Couns Psychother. 2009;21:173-89.

26 WHOQOL Group. Development of the world health organization WHOQOL-BREF Quality of life assessment. Soc sci Med. 1998;15:551-8.
27 Min SK, Lee CI, Kim KI, Suh SY, Kim DK. Development of Korean version of WHO quality of life scale abbreviated version (WHOQOLBREF). J Korean Neuropsychiatr Assoc. 2000;39:571-9.

28 Levy RL, Olden KW, Naliboff BD, Bradley LA, Francisconi C, Drossman DA, Creed F. Psychosocial aspects of the functional gastrointestinal disorders. Gastroenterology. 2006;130:1447-58.

29 Goh KL, Wong HT, Lim CH, Rosaida MS. Time trends in peptic ulcer, erosive reflux esophagitis, gastric and esophageal cancers in a multiracial Asian population. Aliment Pharmacol Ther. 2009;29:774 80.

30 Lien HC, Chang CS, Yeh HZ. Increasing prevalence of erosive esophagitis among Taiwanese aged 40 years and above: a comparison between two time periods. J Clin Gastroenterol. 2009;43:926-32.

31 Kim JI, Kim SG, Kim N. Changing prevalence of upper gastrointestinal disease in 28893 Koreans from 1995 to 2005. Eur J Gastroenterol Hepatol. 2009;21:787-93.

32 Tubbs RL. Occuaptional noise exposure and hearing loss in fire fighters assigned to airport fire stations. Am Ind Hyg Assoc J. 1991;52:372-8.

33 Reichelt PA, Conrad KM. Musculoskeletal injury: ergonomics and physical fitness in firefighters. Occup Med. 1995;10:735-46.

34 Myong JP, Yim HW, Kim HR, Chae JM, Jung YK, Park JI. Depression symptom features of an fire station workers by job. Korean J Occup Health. 2007;46:85-94.

35 Achem SR, DeVault KR. Gastroesophageal reflux disease and the elderly. Gastroenterol Clin North Am. 2014;43(1):147-60.

36 Jansson C, Nordenstedt H, Wallander MA, Johansson S, Johnsen R, Hveem K, et al. Severe gastroesophageal reflux symptoms in relation to anxiety, depression and coping in a population-based study. Aliment Pharmacol Ther. 2007;26:683-91.

37 Hartono JL, Mahadeva S, Goh KL. Anxiety and depression in various functional gastrointestinal disorders: do differences exist? J Dig Dis. 2012;13:252-7.

38 Martin-Merino E, Ruigomez A, Garcia Rodriguez LA, Wallander MA, Johansson S. Depression and treatment with antidepressants are associated with the development of gastroesophageal reflux disease. Aliment Pharmacol Ther. 2010;31:1132-40.

39 Lee DS, Kim JH. Effect of job stress on psychiatric symptoms-focused on gender difference of mediation effect of self-esteem. J korean Neuropsychiatr Assoc. 2001;40(2):217-29.

40 Avidan B, Sonnenberg A, Giblovich H, Sontag SJ. Reflux symptoms are associated with psychiatric disease. Aliment Pharmacol Ther. 2001;15:1907-12.

41 Rubin J, Nagler R, Spiro HM, Pilot ML. Measuring the effect of emotions on esophageal motility. Psychol Med. 1962;24:170-6.

42 Baker LH, Lieberman D, Oehlke M. Psychological distress in patients with gastroesophageal reflux disease. Am J Gastroenterol. 1995; $90: 1797-803$

43 Wallander MA, Jansson C, Johansson S, Johnsen R, Hveem K. Stressful psychosocial factors and symptoms of gastroesophageal reflux disease; a population-based study in Norway. Scand J Gastroenterol. 2010;45:21-9.

44 Masoumi SJ, Khademolhosseini F, Mehrabani D, Moradi F, Mostaghni AA, Zare N, et al. Correlation of quality of life with gastroesophageal reflux disease amongst Qashqai nomads in Iran. Arch Iran Med. 2012; 15:747-50

45 Kovács Z, Seres G, Kerékgyártó O, Czobor P. Psychopathological symptom dimensions in patients with gastrointestinal disorders. J Clin Psychol Med Settings. 2010;17:378-86. 